\title{
Efficacy of sequential methotrexate and 5-fluorouracil (MTX/5FU) in improving oral intake in patients with advanced gastric cancer with severe peritoneal dissemination
}

\author{
Masako Imazawa, Takashi Kojima, Narikazu Boku, Yusuke Onozawa, Shuichi Hironaka, Akira Fukutomi, \\ Hirofumi Yasui, Kentaro Yamazaki, and Keisei Taku \\ Division of Gastrointestinal Oncology, Shizuoka Cancer Center, 1007 Shimonagakubo, Nagaizumi-cho, Shizuoka 411-8777, Japan
}

\begin{abstract}
Background. Although peritoneal dissemination of gastric cancer is common and often causes deterioration of the patient's condition and quality of life (QOL), these patients are usually excluded from clinical trials. We retrospectively investigated the clinical benefit and toxicity of sequential methotrexate and 5-fluorouracil (MTX/5FU) therapy for patients with peritoneal dissemination.

Methods. The subjects were 31 patients with severe peritoneal dissemination of gastric cancer who were treated with MTX/5FU. The treatment schedule comprised weekly administration of MTX $\left(100 \mathrm{mg} / \mathrm{m}^{2}\right)$ followed by $5 F U\left(600 \mathrm{mg} / \mathrm{m}^{2}\right)$. Leucovorin $\left(10 \mathrm{mg} / \mathrm{m}^{2}\right)$ was administered six times, every $6 \mathrm{~h}$, starting $24 \mathrm{~h}$ after MTX administration.

Results. The median survival time was 255 days, and the median progression-free survival was 127 days. Of the 21 patients with measurable lesions, $4(19 \%)$ patients achieved a partial response. Ascites volume decreased markedly in 14 (54\%) of the 26 patients with ascites. Seventeen patients had adequate oral intake, but the other 14 patients had required nutritional support before treatment. The median dripinfusion free survival was 100 days in the former 17 patients, and oral intake improved in $3(21 \%)$ of the latter 14 patients. Grade 3 or 4 neutropenia was observed in $26 \%$ of the patients and anemia was observed in $45 \%$. The grade 3 nonhematological toxicities were vomiting (6\%) and fatigue (10\%). Early death, within 30 days of the last administration of MTX/5FU, occurred due to disease progression in 2 patients, but there were no treatment-related deaths.

Conclusion. MTX/5FU chemotherapy may be effective in treating peritoneal dissemination of gastric cancer and might improve the patient's condition in terms of reducing ascites and improving oral intake.
\end{abstract}

Key words Gastric cancer - Peritoneal dissemination · Sequential MTX/5FU therapy · Oral intake

Offprint requests to: T. Kojima

Received: March 25, 2009 / Aceepted: July 21, 2009

\section{Introduction}

About 50000 deaths from gastric cancer occurred in Japan in 2003 [1], and gastric cancer remains a major cause of cancer deaths in Japan. Although gastric cancer is potentially curable by surgery in the early stages, the prognosis of patients with unresectable or recurrent disease is very poor, and their median survival time is about 3 months when they are treated by best supportive care. Chemotherapy has been shown to improve the survival of these patients significantly [2-4]. More recently, randomized trials performed in various regions of the world have reached some conclusions regarding standard chemotherapy for unresectable or recurrent cancer. However, patients without measurable lesions, such as those suffering only from peritoneal metastasis, were often excluded from such clinical trials in the West; this patient group has been neglected, because most trials have considered the response rate as one of the important parameters for evaluation. Thus, data regarding an optimal therapy for this population remains sparse.

In Japan, a combination of S-1 (an oral formulation of tegafur, 5-chloro-24-dyhydropyridine, and potassium oxonate, at a molar ratio of 1:0.4:1) and cisplatin has been recognized as a standard of care after the results of two randomized trials, Japan Clinical Oncology Group (JCOG) 9912 [5] and S-1 Plus cisplatin versus S-1 In RCT In the Treatment for Stomach cancer (SPIRITS) trial [6]. Patients without a measurable lesion had been eligible to enter these trials and those with mild peritoneal metastasis were actually shown by a subset analysis to have benefited from the combination therapy. However, patients with severe peritoneal metastasis with clinical symptoms such as massive ascites and bowel obstruction had been ineligible for both the trials, because patients had to be treated by oral agents in at least one of the treatment arms.

Treatment with sequential methotrexate and 5fluorouracil (MTX/5FU) has been widely used in Japan 
to treat gastric cancer patients, particularly those with peritoneal metastasis. Although a phase II study to test its efficacy (JCOG 9207) had shown a disappointing response rate of $9.0 \%$ [7], this trial included only pretreated patients with measurable disease. The same study group launched another phase II trial in which only gastric cancer patients with malignant ascites were eligible (JCOG 9603) and, despite the overall response rate of only $5.7 \%$ among patients who had measurable disease, clinical response against the ascites was shown in $35.1 \%$ of the patients [8]. This finding, along with the findings of another, retrospective, study from the same country in which malignant ascites disappeared completely in 5 of 26 patients [9], suggests the benefit of MTX/5FU for patients with peritoneal carcinomatosis.

In the present study, the efficacy and toxicity of MTX/5FU were evaluated in previously untreated gastric cancer patients with severe peritoneal metastasis. In addition, the study focused on survival time without drip infusion for nutritional support, because this is considered to be a clinically relevant endpoint in patients who suffer from peritoneal carcinomatosis and are ultimately incurable.

\section{Subjects and methods}

\section{Subjects}

The subjects were recruited from patients who received MTX/5FU therapy at Shizuoka Cancer Center between October 2002 and August 2006, according to the following criteria: (1) histologically confirmed gastric adenocarcinoma; (2) unresectable or recurrent disease; (3) severe peritoneal dissemination with bowel stenosis confirmed by barium enema and/or ascites beyond the pelvic cavity detected by computed tomography (CT) scan; (4) age 20-75 years; (5) performance status (PS) 2 or less on the Eastern Cooperative Oncology Group (ECOG) scale; (6) no history of chemotherapy or radiation therapy; (7) white blood cell count between 3000 and $10000 / \mathrm{mm}^{3} ;(8)$ platelet count $100000 / \mathrm{mm}^{3}$ or more; (9) adequate liver function, as indicated by serum concentrations of bilirubin $2.0 \mathrm{mg} / \mathrm{dl}$ or less, aspartate aminotransferase $100 \mathrm{IU} / \mathrm{l}$ or less, and alanine aminotransferase $100 \mathrm{IU} / 1$ or less; (10) serum creatinine concentration $1.5 \mathrm{mg} / \mathrm{dl}$ or less; and (11) no blood transfusion within 14 days before the start of chemotherapy. The presence of a measurable metastatic lesion was not mandatory. Patients with active bleeding from the gastrointestinal tract, ileus requiring insertion of an ileus tube, other active synchronous carcinoma, central nervous system metastasis or concurrent uncontrolled medical illness, and massive ascites requiring drainage were excluded.

\section{Treatment schedule}

The treatment schedule comprised weekly administration of MTX $\left(100 \mathrm{mg} / \mathrm{m}^{2}\right.$, i.v. bolus) followed by $5 \mathrm{FU}$ $\left(600 \mathrm{mg} / \mathrm{m}^{2}\right.$, i.v. bolus) after a 3 -h interval. Leucovorin rescue $\left(10 \mathrm{mg} / \mathrm{m}^{2}\right.$ p.o. or i.v. every $6 \mathrm{~h}$, six times) was commenced $24 \mathrm{~h}$ after MTX administration. To prevent toxicity from MTX, patients were hydrated with $500 \mathrm{ml}$ of solution containing sodium bicarbonate $(33.3 \mathrm{mEq})$ during the interval between the administration of MTX and $5 \mathrm{FU}$, and acetazolamide $(250 \mathrm{mg})$ was given intravenously immediately after the infusion of MTX, for urine alkalinization. Treatment was repeated until disease progression, development of unacceptable toxicity, or the patient's refusal to continue. When grade 4 hematological or grade 3 or 4 nonhematological toxicity occurred, or when the attending physician judged it appropriate, the dose of MTX and 5FU was reduced to $80 \%$. Once serious toxicity was observed, treatment was suspended until recovery.

\section{Response and toxicity evaluation}

Objective responses of measurable metastatic lesions were evaluated according to the response evaluation criteria in solid tumors (RECIST) guideline. The tumor response was evaluated using a CT scan every 4-8 weeks after the initiation of treatment. A nontarget lesion was defined as a lesion smaller than $1 \mathrm{~cm}$ at the primary site, bone metastasis, pleural effusion, or ascites. Progressive disease (PD) was defined as the unequivocal progression of nontarget lesions, the appearance of any new lesions, or deterioration in clinical status that was considered to have been caused by disease progression.

Toxicity was monitored weekly and evaluated according to the common terminology criteria for adverse events, version 3.0 JCOG/JSCO (Japan Society of Clinical Oncology) (CTCAE ver.3 JCOG/JSCO) [10]. The response of ascites was evaluated using an abdominal CT scan, based on the following criteria specified in the Japanese classification of gastric carcinoma (13th edition) [11]: (1) complete response (CR), complete disappearance of ascites for 4 weeks, confirmed by CT scan; (2) partial response (PR), dramatic decrease in ascites volume for 4 weeks, confirmed by CT scan; (3) no response, other than criteria (1) or (2) including an unequivocal increase in ascites.

\section{Statistical analysis}

Overall survival was calculated from the initiation of treatment to the date of death or the last follow-up day in survivors. Progression-free survival was calculated from the initiation of treatment to the date of detection of disease progression or death from any cause. In patients who had not required nutritional support before 
chemotherapy, drip-infusion-free survival was calculated from the initiation of treatment to the date when drip infusion for nutritional support was first started. The oral intake of patients who had initially required nutritional support was considered to have improved when nutritional support could be stopped for at least 1 week. Overall survival, progression-free survival, and the drip-infusion-free survival were calculated using the Kaplan-Meier method and the StatView version 5.0.1 software program (SAS Institute, Cary, NC, USA).

\section{Results}

\section{Patients' characteristics and treatment}

Between October 2002 and August 2006, 76 patients with unresectable or recurrent gastric cancer received MTX/5FU at our hospital. Forty-five patients were excluded, for the following reasons: absence of peritoneal metastasis $(n=7)$, poor PS $(n=7)$, age more than 75 years $(n=3)$, disseminated intravascular coagulation $(n=3)$, afferent loop syndrome $(n=2)$, ileus $(n=1)$, cardiac tamponade $(n=1)$, respiratory failure due to lymphangitis carcinomatosa $(n=1)$, deep venous thrombosis $(n=2)$, another active malignancy $(n=1)$, liver dysfunction $(n=1)$, massive ascites requiring drainage $(n=11)$, massive pleural effusion $(n=2)$, blood transfusion $(n=1)$, and loss to follow up $(n=2)$.

The remaining 31 patients were the subjects of this study. Their characteristics are shown in Table 1. The median age was 62 years (range, 25-74 years). A high percentage of patients $(84 \%)$ had a good PS of 0 or 1 . Twenty-three patients had histologically diffuse-type adenocarcinoma (poorly differentiated adenocarcinoma in 11 , signet ring cell carcinoma in 11 , mucinous carcinoma in 1 . All patients were assessable for overall survival, progression-free survival, and toxicity. Twenty-one patients had at least one measurable lesion whose objective responses were assessable. Twenty-six patients had ascites whose responses were assessable. Drip-infusionfree survival could be assessed in 17 patients who had adequate oral intake, and 14 patients who had received nutritional support by total parental nutrition before treatment were assessable for improved oral intake.

\section{Treatment course}

In total, 557 administrations of sequential MTX/5FU were performed in 31 patients. The median number of administrations was 17 (range, 2-57). The median follow-up period was 496 days in survivors at the time of analysis. The dose was reduced in two patients; one patient had experienced grade 3 fatigue, and the other patient had experienced grade 4 neutropenia. One patient was treated with a three-times-weekly adminis-
Table 1. Patients' characteristics $(n=31)$

\begin{tabular}{|c|c|}
\hline Characteristic & \\
\hline Sex & \\
\hline Male & 15 \\
\hline Female & 16 \\
\hline Age, years, median (range) & $62(25-74)$ \\
\hline ECOG performance status & \\
\hline 0 & 10 \\
\hline 1 & 16 \\
\hline 2 & 5 \\
\hline Primary tumor & \\
\hline Yes & 23 \\
\hline No & 8 \\
\hline Macroscopic type (Japanese & \\
\hline 0 & 2 \\
\hline 1 & 1 \\
\hline 2 & 4 \\
\hline 3 & 14 \\
\hline 4 & 9 \\
\hline Unknown & 1 \\
\hline Histological type & \\
\hline Intestinal type & 8 \\
\hline Diffuse type & 23 \\
\hline Metastatic sites & \\
\hline Lymph nodes & 18 \\
\hline Ovary & 3 \\
\hline Liver & 2 \\
\hline Bone & 2 \\
\hline Adrenal gland & 1 \\
\hline Muscle & 1 \\
\hline Number of metastatic sites & \\
\hline 1 & 10 \\
\hline 2 & 16 \\
\hline 3 & 4 \\
\hline 4 & 1 \\
\hline Target lesion & \\
\hline Yes & 21 \\
\hline No & 10 \\
\hline Ascites & \\
\hline Yes & 26 \\
\hline No & 5 \\
\hline Oral intake & \\
\hline Possible & 17 \\
\hline Impossible & 14 \\
\hline
\end{tabular}

ECOG, Eastern Cooperative Oncology Group

tration and 1-week-off schedule because of grade 2 diarrhea and appetite loss. The other patient requested and received a biweekly treatment schedule. The most frequent reason for treatment termination was disease progression (27 patients; $87 \%$ ). In one patient treatment was terminated because of severe toxicity: grade 4 increase in serum creatinine concentration.

\section{Toxicity}

The worst grades of toxicity per patient during the treatment course are summarized in Table 2. Anemia was the most common hematological toxicity, and 14 of the 31 patients $(45 \%)$ experienced grade 3 or 4 . Grade 3 or 4 neutropenia occurred in $26 \%$ of the patients and grade 3 
Table 2. Toxicity profiles $(n=31)$

\begin{tabular}{lccc}
\hline & $\begin{array}{c}\text { Grade (NCI-CTC, } \\
\text { version 3.0; } \%)\end{array}$ & \\
\cline { 2 - 3 } Toxicity & 3 & 4 & $3 / 4(\%)$ \\
\hline Hematological & & & \\
$\quad$ Leukopenia & 6 & 3 & 29 \\
$\quad$ Neutropenia & 7 & 1 & 26 \\
Anemia & 9 & 5 & 45 \\
Thrombocytopenia & 2 & 0 & 6 \\
Nonhematological & & & \\
Nausea & 0 & 0 & 0 \\
$\quad$ Vomiting & 2 & 0 & 6 \\
$\quad$ Diarrhea & 1 & 0 & 3 \\
$\quad$ Fatigue & 3 & 0 & 10 \\
Anorexia & 1 & 0 & 3 \\
Mucositis & 1 & 0 & 3 \\
Rash & 1 & 0 & 3 \\
\hline
\end{tabular}

Two patients $(6 \%)$ died within 30 days of the last methotrexate/5fluorouracil (MTX/5FU) chemotherapy administration

NCI-CTC, National Cancer Institute common toxicity criteria

or 4 leukopenia in $29 \%$. Grade 3 nonhematological toxicities were vomiting $(6 \%)$, fatigue $(10 \%)$, anorexia $(3 \%)$, mucositis $(3 \%)$, and rash $(3 \%)$. One patient experienced a grade 4 increase in serum creatinine concentration after six courses of the treatment; this increase resolved after appropriate treatment. Early death within 30 days from the last administration of MTX/5FU occurred in two patients. One patient died of severe aspiration pneumonia caused by ileus due to disease progression 13 days after treatment. The other patient died on day 16 after changing to another hospital, and detailed information was not available after the change.

\section{Efficacy}

The responses to treatment and responses of ascites are shown in Table 3. No patients achieved a CR, and 4 patients achieved a PR, with a response rate of $19 \%$ (95\% confidence interval [CI], 2.25\%-35.84\%). Objective improvement of ascites was seen in 14 of 26 patients $(54 \%)$. The overall survival is shown in Fig. 1 . The median survival time was 255 days, and the median progression-free survival was 127 days. The dripinfusion-free survival of the 17 patients with initially adequate oral intake is shown in Fig. 2. The median drip-infusion-free survival time was 100 days. The rate of improvement in oral intake in the 14 patients who initially required nutritional support was $21 \%$ (3 of 14 ). In these 3 patients, the drip-infusion-free durations were 9 days, 118 days, and 145 days, respectively.

\section{Discussion}

In clinical practice, the clinician must select an optimal regimen for each patient based on the medical condition
Table 3. Response to treatment

\begin{tabular}{lc}
\hline Response & Number of patients (\%) \\
\hline Assessable for response $(n=21)$ & $21(68)$ \\
Objective response & \\
PR & $4(19)$ \\
SD & $12(57)$ \\
PD & $5(24)$ \\
Response rate & $4(19)$ \\
Response of ascites $(n=26)$ & \\
Assessable for response & $26(84)$ \\
CR & $10(38)$ \\
PR & $4(15)$ \\
No response & $12(46)$ \\
Response rate & $14(54)$
\end{tabular}

$\mathrm{PR}$, partial response; $\mathrm{SD}$, stable disease; $\mathrm{PD}$, progressive disease; $\mathrm{CR}$, complete response

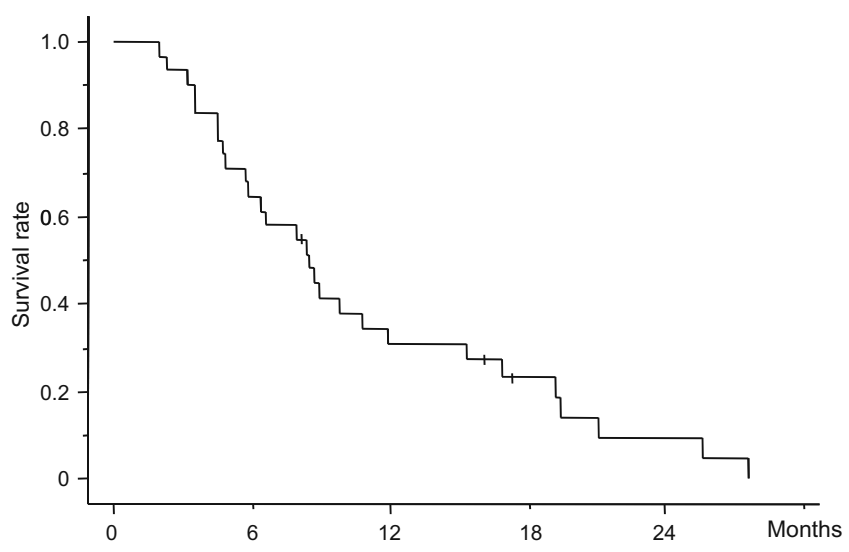

Fig. 1. Overall survival

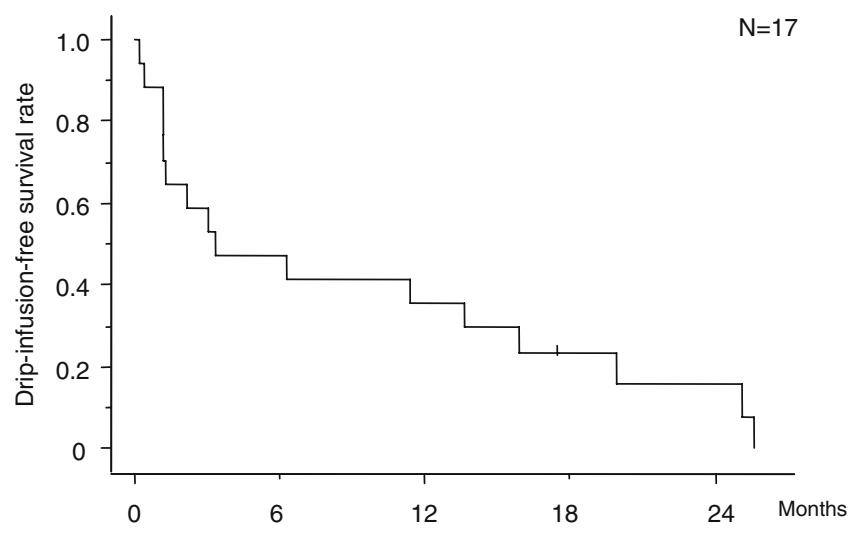

Fig. 2. Drip-infusion-free survival of the patients with initially adequate oral intake

and other factors such as age, performance status (PS), oral intake, organ functions, and extent of the disease. Cisplatin-based regimens are usually considered inappropriate for patients with severe peritoneal dissemination and retention of ascites, because these patients 
have potential renal impairment or poor PS, which makes it difficult for them to tolerate the large-volume hydration needed to prevent cisplatin-induced renal injury. Oral agents such as S-1 are also not suitable.

Many studies have suggested that MTX/5FU is effective in patients with advanced gastric cancer. We found a $19 \%$ response rate, disease stabilization in $76 \%$ of the patients with measurable metastatic lesions, and 54\% response rate of ascites. The median survival time was about 8.5 months. These results are similar to those of previous reports and support the notion that MTX/5FU is effective for patients with peritoneal dissemination and that this treatment improves oral intake.

With regard to toxicity, $26 \%$ of patients experienced grade 3 or 4 neutropenia, and $10 \%$ experienced grade 3 fatigue. These results suggest that MTX/5FU has substantial toxicities in patients with severe peritoneal dissemination. Although we observed no treatment-related deaths, there were two early deaths caused by disease progression. We recommend that when using MTX/5FU to treat patients with peritoneal dissemination from gastric cancer, the clinician should be alert to the signs of toxicity and to the sudden deterioration in a patient's medical condition because of disease progression.

Patients with peritoneal dissemination may have difficulty maintaining adequate oral intake during disease progression because of intestinal obstruction or massive ascites, and nutritional support is indicated. Previous reports showed rates of improvement in oral intake of $29 \%-38 \%$ with MTX/5FU regimens $[8,9]$. Three of our 14 patients $(21 \%)$ who had required nutritional support before the treatment achieved improved oral intake. The median drip-infusion-free survival time in the present study was 100 days. Tahara et al. [9] reported a median drip-infusion-free survival of 178 days. Yamao et al. [8], in reporting the results of JCOG 9603, did not report the rates of improvement in oral intake, or the data for drip-infusion-free survival. Oral intake is important for quality of life (QOL), and these results suggest that MTX/5FU chemotherapy can improve and maintain QOL in patients with peritoneal dissemination from gastric cancer. However, our study was retrospective and its sample size was small. The indication for nutritional support depends on the physician's judgment and the patient's background and requests. Differences in these factors may explain the differences in rates of improvement in oral intake and drip-infusionfree survival between our study and previous reports. The efficacy of MTX/5FU should ultimately be investigated in a phase III trial with a sufficient sample size. In fact, a randomized study comparing continuous $5 \mathrm{FU}$ infusion with MTX/5FU in gastric cancer patients with clinically apparent peritoneal metastasis (JCOG 0106) has completed accrual and awaits final survival analysis. It is expected that this trial will establish a tentative standard of care for this population. However, patients with severe peritoneal metastasis generally suffer from short life expectancy and such patients were often found to be unsuitable for registration even for the JCOG 0106 study. In the present study, the analysis of a consecutive series of patients implies that even those with severe peritoneal disease could benefit from chemotherapy, provided that they had not been pretreated and that the utmost care is taken to manage adverse events.

In conclusion, MTX/5FU chemotherapy has potential for improving and maintaining oral intake in patients with severe peritoneal dissemination from gastric cancer.

\section{References}

1. Statistics and Information Department, Minister's Secretariat, Ministry of Health, Labour and Welfare. Vital statistics of Japan (in Japanese). Tokyo: Ministry of Health, Labour and Welfare.

2. Murad AM, Santiago FF, Petroianu A, Rocha PR, Rodrigues MA, Rausch M. Modified therapy with 5-fluorouracil, doxorubicin, and methotrexate in advanced gastric cancer. Cancer 1993; 72:37-41.

3. Pyrhonen S, Kuitunen T, Nyandoto P, Kouri M. Randomised comparison of fluorouracil, epidoxorubicin and methotrexate (FEMTX) plus supportive care with supportive care alone in patients with nonresectable gastric cancer. Br J Cancer 1995;71: 587-91.

4. Glimelius B, Hoffman K, Haglund U, Nyrén O, Sjödén PO. Initial or delayed chemotherapy with best supportive care in advanced gastric cancer. Ann Oncol 1994;5:189-90.

5. Boku N, Yamamoto S, Shirao K, Doi T, Sawaki A, Koizumi W, et al. Randomized phase III study of 5-fluorouracil (5-FU) alone versus combination of irinotecan and cisplatin (CP) versus S-1 alone in advanced gastric cancer (JCOG9912). J Clin Oncol 2007 ASCO Annual Meeting Proceedings Part I;25:18S(June 20 Supplement):LBA4513.

6. Koizumi W, Narahara H, Hara T, Takagane A, Akiya T, Takagi $\mathrm{M}$, et al. S-1 plus cisplatin versus S-1 alone for first-line treatment of advanced gastric cancer (SPIRITS trial): a phase III trial. Lancet Oncol 2008;9:215-21.

7. Hamaguchi T, Shirao K, Yamamichi N, Hyodo I, Koizumi W, Seki S, et al. A Phase II Study of Sequential Methotrexate and 5-fluorouracil chemotherapy in previously treated gastric cancer: a report from the Gastrointestinal Oncology Group of the Japan Clinical Oncology Group, JCOG 9207 Trial. Jpn J Clin Oncol 2008;38:432-7.

8. Yamao T, Shimada Y, Shirao K, Ohtsu A, Ikeda N, Hyodo I, et al. Phase II study of sequential methotrexate and 5-fluorouracil chemotherapy against peritoneally disseminated gastric cancer with malignant ascites: a report from the Gastrointestinal Oncology Study Group of the Japan Clinical Oncology Group, JCOG 9603 Trail. Jpn J Clin Oncol 2004;34:316-22.

9. Tahara M, Ohtsu A, Boku N, Nagashima F, Myto M, Sano Y, et al. Sequential methotrexate and 5-fluorouracil therapy for gastric cancer patients with peritoneal dissemination: a retrospective study. Gastric Cancer 2001;4:212-8.

10. Anonymous. Japanese translation of common terminology criteria for adverse events (CTCAE), and instructions and guidelines. Int J Clin Oncol 2004;9:1-82.

11. Japanese Gastric Cancer Association. Japanese Classification of Gastric Carcinoma - 2nd English edition - Response assessment of chemotherapy and radiotherapy for gastric carcinoma: clinical criteria. Gastric Cancer 2001;4:1-8. 